\title{
Sugarcane Family Selection and Genetic Parameter Prediction via the REML/BLUP Methodology
}

\author{
Hugo Zeni Neto ${ }^{1}$, Renato Frederico dos Santos ${ }^{1}$, Luiz Gustavo da Mata Borsuk ${ }^{1}$, Henrique Sanches Angeli ${ }^{1}$ \\ $\&$ Guilherme Souza Berton ${ }^{2}$ \\ ${ }^{1}$ Agronomy Department, Maringá State University, Maringá, PR, Brazil \\ ${ }^{2}$ Agronomy Department, Parana Federal University, Curitiba, PR, Brazil \\ Correspondence: Renato Frederico dos Santos, Agronomy Department, Maringá State University, Colombo \\ Avenue, n.5790-Bloco J45, 87020-900, Maringá, Paraná State, Brazil. Tel: 55-449-8853-7271. E-mail: \\ agrorfs@hotmail.com
}

Received: April 14, 2019

Accepted: May 21, 2019 Online Published: July 31, 2019

doi:10.5539/jas.v11n11p303

URL: https://doi.org/10.5539/jas.v11n11p303

\begin{abstract}
Most sugarcane breeding programs tend to evaluate low heritability characteristics during the initial stages of genotype selection. Thus, family selection has been recently preferred. In this context, the aim of the present study was to select the best family among 78 sugarcane families, as well as estimate genetic values through the mixed models of restricted-maximum likelihood and best non-bias predictor (REML/BLUP) methodology, originating from the República Brasil 2005 (RB05) series. This strategy was deemed efficient, and 34 to 38 families were chosen from four evaluated characteristics underexplored by genetic researchers such as total plot mass (MTT), mean mass of one tiller in the plot (M1C), stature (EST), and mean number of canes per square meter (NCM). The family increments ranging from 6.02 to $82.11 \%$, in the next genetic culture improvement program selection phases.
\end{abstract}

Keywords: likelihood, mixed model, Saccharum spp., sugarcane breeding

\section{Introduction}

Due to Brazil's privileged position regarding sugarcane (Saccharum spp.) cultivation, crop breeding programs have focused on developing new cultivars with high productive sugarcane and sugar potential. In this context, it is crucial to select materials during the early stages of the breeding program. Usually, low heritability characteristics are evaluated during these initial stages. However, a high number of individuals is generally selected, as these characteristics present high coefficients of variation. To avoid this, the family selection procedure is adopted, which aims to select the best sugarcane families and reject the worst (Silva, Barbosa, Resende, Peternelli, \& Pedrozo, 2015), since the best families tend to be more effective in generate promising clones for the next breeding program phase.

Family selection has been previously reported in other studies, due to its crucial importance in increasing genetic gain in breeding programs, not only by measuring an individual's potential to be a future genitor, but also in predicting potential values. This allows for strategy alterations in the next selection steps, if necessary, increasing experiment efficiencies and estimating the additive variance of the plants (Brasileiro, Paula Mendes, Peternelli, Silveira, Resende, \& Barbosa, 2016; Almeida, Viana, Amaral Júnior, \& Júnior, 2014; Atkin, Dieters, \& Stringer, 2009).

Among family selection methodologies, REML/BLUP is the most noteworthy (Meyer, 1991; Resende, 2002). BLUP has been routinely applied in animal breeding and, recently, in plant breeding programs (Almeida Filho, Tardin, Guimarães, \& Resende, 2016; Oakey, Verbyla, Pitchford, Cullis, \& Kuchel, 2006; Oakey, Verbyla, Cullis, Wei, \& Pitchford, 2007), as it is more precise than other methodologies, such as AMMI, for example, as it applies genotype correlation information from individuals. REML exhibits certain advantages compared to the least squares (ordinary or generalized) method (Kennedy \& Sorensen, 1988), as it applies information regarding the individuals themselves (or from the genitors, if necessary), making it unbiased. In addition, it also takes into account the genetic covariance among treatments, weighing genotype imbalances within the adopted statistical design. 
Variable responses regarding heritability in the narrow sense, from low to medium magnitude, are available in the literature, including clump mass, ${ }^{\circ}$ Brix, tons of cane per hectare and average mass of the tiller, among others, which fit mixed models and family selection methodologies (Castro, Peternelli, Resende, Marinho, Costa, Barbosa, \& Moreira, 2016; Oliveira et al., 2008; Pedroso et al., 2009).

In this context, the aim of the present study is to select genetic materials by applying the RELM/BLUP methodology for the RB05 series from the Sugarcane Genetic Improvement Program (PMGCA), from the Inter-University Network for the Development of the Sugaralcohol sector (RIDESA), in its first improvement phase (T1). Genetic parameters for four underexplored sugarcane characteristics, namely total plot mass (MTT), mean mass of one tiller in the plot (M1C), stature (EST), and mean number of canes per square meter (NCM) where also evaluated.

\section{Material and Methods}

The field experiment comprised a total of biparental cross 78 families (Table 1) (a cross where the male and female parents are known), in a Federer block arrangement (Federer, 1956) with five replicates, where each experimental plot was considered as being one clump of sugarcane, planted in two $5 \mathrm{~m}$ rows, with a $1.40 \mathrm{~m}$ space between rows and $0.50 \mathrm{~m}$ between individuals, in the municipality of Paranavaí $\left(22^{\circ} 58^{\prime} \mathrm{S}, 52^{\circ} 28^{\prime} \mathrm{W}, 503\right.$ $\mathrm{m}$ a.s.1.), in the state of Paraná (PR), Brazil. 
Table 1. Number of biparental crosses, relationship between female and male parents and codes used to identify the crosses used herein regarding the study of Saccharum spp. sibling species families from the RB05, Paranavai municipality, Paraná, Brazil

\begin{tabular}{|c|c|c|c|c|c|c|c|}
\hline $\mathrm{N}^{\mathrm{o}}$ & Female & Male & Family Code & $\mathrm{N}^{\mathrm{o}}$ & Female & Male & Family Code \\
\hline 1 & Co434 & RB946915 & F23M76 & 40 & RB855511 & RB961530 & F01M39 \\
\hline 2 & Co775 & RB855035 & F27M56 & 41 & RB931604 & RB957751 & F19M29 \\
\hline 3 & Laica98-208 & RB855035 & F32M56 & 42 & RB945961 & RB956911 & F10M51 \\
\hline 4 & RB945956 & RB855035 & F70M56 & 43 & RB945961 & RB957751 & F10M29 \\
\hline 5 & RB855035 & RB945956 & F56M70 & 44 & RB92606 & RB971537 & F04M40 \\
\hline 6 & RB945956 & IAC87-3396 & F70M30 & 45 & RB92606 & RB971551 & F04M79 \\
\hline 7 & RB945956 & RB945065 & F70M75 & 46 & RB971537 & RB943339 & F40M74 \\
\hline 8 & RB945956 & RB947501 & F70M48 & 47 & RB943339 & RB971537 & F74M40 \\
\hline 9 & RB945065 & RB945956 & F75M70 & 48 & IAC93-7009 & H83-9998 & F11M69 \\
\hline 10 & RB947501 & RB945956 & F48M70 & 49 & RB71114 & SP91-1049 & F44M02 \\
\hline 11 & RB941531 & IAC 87-3396 & F66M30 & 50 & RB855563 & SP91-1049 & F34M02 \\
\hline 12 & IAC87-3396 & RB855063 & F30M36 & 51 & RB896342 & RB961527 & F26M55 \\
\hline 13 & RB912695 & RB945065 & F49M75 & 52 & RB896342 & RB92508 & F26M65 \\
\hline 14 & RB947501 & SP80-3280 & F48M41 & 53 & RB915141 & RB855322 & F57M12 \\
\hline 15 & RB739735 & SP80-3280 & F63M41 & 54 & RB91537 & SP91-1049 & F81M02 \\
\hline 16 & SP80-3280 & RB947501 & F41M48 & 55 & RB925211 & SP70-1143 & F67M37 \\
\hline 17 & SP80-3280 & L60-14 & F41M22 & 56 & RB925211 & SP91-1049 & F67M02 \\
\hline 18 & SP80-3280 & RB72454 & F41M82 & 57 & RB925345 & RB915124 & F54M07 \\
\hline 19 & SP80-3280 & RB835486 & F41M60 & 58 & RB93522 & RB957689 & F35M06 \\
\hline 20 & SP80-3280 & RB867515 & F41M64 & 59 & RB936001 & RB965586 & F45M78 \\
\hline 21 & SP80-3280 & RB872552 & F41M21 & 60 & RB945962 & RB947532 & F59M18 \\
\hline 22 & SP80-3280 & RB965911 & F41M20 & 61 & RB945962 & RB9620 & F59M50 \\
\hline 23 & SP80-3280 & SP70-1284 & F41M53 & 62 & RB945964 & SP91-1049 & F42M02 \\
\hline 24 & SP80-3280 & SP71-6949 & F41M47 & 63 & RB946022 & RB925211 & F09M67 \\
\hline 25 & SP83-2847 & L60-14 & F14M22 & 64 & RB951015 & RB957712 & F13M17 \\
\hline 26 & RB72454 & RB855511 & F82M01 & 65 & RB915141 & SP89-1115 & F57M46 \\
\hline 27 & RB9557 & RB72454 & F80M82 & 66 & RB957610 & RB93522 & F62M35 \\
\hline 28 & SP83-2847 & RB72454 & F14M82 & 67 & RB957712 & RB93522 & F17M35 \\
\hline 29 & RB835486 & IAC86-2210 & F60M16 & 68 & RB957712 & RB945954 & $\mathrm{F} 17 \mathrm{M} 25$ \\
\hline 30 & RB835486 & RB835089 & F60M52 & 69 & RB962002 & RB965921 & F03M33 \\
\hline 31 & RB835486 & RB855127 & F60M43 & 70 & RB965586 & RB936001 & F78M45 \\
\hline 32 & RB835486 & RB931604 & F60M19 & 71 & RB966920 & SP89-1115 & F58M46 \\
\hline 33 & RB835486 & RB945961 & F60M10 & 72 & SP80-1842 & RB83102 & F61M38 \\
\hline 34 & RB835486 & RB961539 & F60M71 & 73 & SP85-3877 & RB961005 & F05M68 \\
\hline 35 & SP71-6949 & CB45-155 & F47M31 & 74 & SP89-1115 & RB735200 & F46M72 \\
\hline 36 & SP83-2847 & RB855206 & F14M08 & 75 & SP89-1115 & RB855536 & F46M73 \\
\hline 37 & RB863129 & SP83-2847 & F15M14 & 76 & SP89-1115 & RB915124 & F46M07 \\
\hline 38 & RB92606 & SP83-2847 & F04M14 & 77 & SP89-1115 & RB935860 & F46M28 \\
\hline 39 & RB855511 & RB855156 & F01M24 & 78 & SP91-1049 & RB9364 & F02M77 \\
\hline
\end{tabular}

The MTT was obtained with the aid of a scale in the field. The sugarcane stem was cut close to the ground and green leaves were discarded and weighed. The M1C was obtained by counting the number of tillers from the experimental plot and the MTT ratio was calculated by counting tillers. EST was measured with a 5-by-5 cm graduated ruler, totaling $4 \mathrm{~m}$, placed in the center of the clump, and height was measured to the first visible dewlap, thus characterizing leaf +1 (Dillewijn, 1952). The ratio for the area was calculated at the planting line intersections $(1.4 \mathrm{~m} \times 5 \mathrm{~m})$, totaling $7 \mathrm{~m}^{2}$, in order to calculate NCM.

The data were analyzed using the software SELEGEN-REML/BLUP (Resende, 2007), under model 35 (Incomplete Blocks and One Plant per Plot, Non-Similar Genitors):

$$
\mathrm{y}=\mathrm{Xr}+\mathrm{Za}+\mathrm{Wf}+\mathrm{Sb}+\mathrm{e}
$$


Where, $\mathrm{y}$ is the data vector, $\mathrm{r}$ is the vector of repetition effects (assumed to be fixed) added to the general means, $\mathrm{a}$ is the vector of individual additive genetic effects (assumed to be random), $\mathrm{f}$ is the vector of family dominance effects of sibling species (assumed to be random), b s the vector of block effects (assumed to be random) and e is the vector of errors or residues (assumed to be random). X, Z, W and S represent the incidence matrices for the effects.

The means and variances of this model present the following distributions and structures:

$$
\begin{aligned}
\mathrm{y} \mid \mathrm{r}, \mathrm{V} & \sim \mathrm{N}(\mathrm{Xr}, \mathrm{V}) \\
\mathrm{a} \mid \mathrm{A} 1 \hat{\sigma}_{\mathrm{a}}^{2} & \sim \mathrm{N}\left(0, \mathrm{~A} 1 \hat{\sigma}_{\mathrm{a}}^{2}\right) \\
\mathrm{f} \mid \mathrm{I} \hat{\sigma}_{\mathrm{f}}^{2} & \sim \mathrm{N}\left(0, \mathrm{I} \hat{\sigma}_{\mathrm{f}}^{2}\right) \\
\mathrm{b} \mid \mathrm{I} \hat{\sigma}_{\mathrm{b}}^{2} & \sim \mathrm{N}\left(0, \mathrm{I} \hat{\sigma}_{\mathrm{b}}^{2}\right) \\
\mathrm{e} \mid \mathrm{I} \hat{\sigma}_{\mathrm{e}}^{2} & \sim \mathrm{N}\left(0, \mathrm{I} \hat{\sigma}_{\mathrm{e}}^{2}\right)
\end{aligned}
$$

How,

$$
\mathrm{E}=\left[\begin{array}{c}
\mathrm{y} \\
\mathrm{a} \\
\mathrm{f} \\
\mathrm{b} \\
\mathrm{e}
\end{array}\right]=\left[\begin{array}{c}
\mathrm{Xr} \\
0 \\
0 \\
0 \\
0
\end{array}\right] \text { and } \operatorname{Var}\left[\begin{array}{c}
\mathrm{y} \\
\mathrm{a} \\
\mathrm{f} \\
\mathrm{b} \\
\mathrm{e}
\end{array}\right]=\left[\begin{array}{ccccc}
\mathrm{V} & \mathrm{ZA} \sigma_{\mathrm{a}}^{2} & \mathrm{WI} \sigma_{\mathrm{f}}^{2} & \mathrm{SI} \sigma_{\mathrm{b}}^{2} & \mathrm{I} \sigma_{\mathrm{e}}^{2} \\
\mathrm{~A} \sigma_{\mathrm{a}}^{2} \mathrm{Z}^{\prime} & \mathrm{A} \sigma_{\mathrm{a}}^{2} & 0 & 0 & 0 \\
\mathrm{I} \sigma_{\mathrm{f}}^{2} \mathrm{~W}^{\prime} & 0 & \mathrm{WI} \sigma_{\mathrm{f}}^{2} & 0 & 0 \\
\mathrm{I} \sigma_{\mathrm{b}}^{2} \mathrm{~S}^{\prime} & 0 & 0 & \mathrm{SI} \sigma_{\mathrm{b}}^{2} & 0 \\
\mathrm{I} \sigma_{\mathrm{e}}^{2} & 0 & 0 & 0 & \mathrm{I} \sigma_{\mathrm{e}}^{2}
\end{array}\right]
$$

So,

$$
\mathrm{V}=\operatorname{Var}(\mathrm{y})=\mathrm{ZA} \hat{\sigma}_{\mathrm{a}}^{2} \mathrm{Z}^{\prime}+\mathrm{WI} \hat{\sigma}_{\mathrm{f}}^{2} \mathrm{~W}^{\prime}+\mathrm{SI} \hat{\sigma}_{\mathrm{b}}^{2} \mathrm{~S}^{\prime}+\mathrm{I} \hat{\sigma}_{\mathrm{e}}^{2}
$$

with $\mathrm{A}$ being the additive genetic correlation matrix among the individuals evaluated in this study. Therefore, $\operatorname{Cov}\left(\mathrm{a}, \mathrm{b}^{\prime}\right)=0 ; \operatorname{Cov}\left(\mathrm{a}, \mathrm{e}^{\prime}\right)=0 ; \operatorname{Cov}\left(\mathrm{f}, \mathrm{b}^{\prime}\right)=0 ; \operatorname{Cov}\left(\mathrm{f}, \mathrm{e}^{\prime}\right)=0$ and $\operatorname{Cov}\left(\mathrm{b}, \mathrm{e}^{\prime}\right)=0$.

The equations of the model were,

$$
\left[\begin{array}{cccc}
X^{\prime} X & X^{\prime} V & X^{\prime} W & X^{\prime} S \\
Z^{\prime} X & Z^{\prime} Z+A^{-1} \lambda_{1} & Z^{\prime} W & Z ' S \\
W^{\prime} X & W^{\prime} Z & W^{\prime} W+I \lambda_{2} & W^{\prime} S \\
S^{\prime} X & S^{\prime} Z & S^{\prime} W & S^{\prime} S+I \lambda_{3}
\end{array}\right]\left[\begin{array}{c}
\hat{r} \\
\hat{a} \\
\hat{f} \\
\hat{b}
\end{array}\right]=\left[\begin{array}{c}
X^{\prime} y \\
Z^{\prime} y \\
W^{\prime} y \\
S^{\prime} y
\end{array}\right]
$$

On what,

$$
\lambda_{1}=\frac{\hat{\sigma}_{\mathrm{e}}^{2}}{\hat{\sigma}_{\mathrm{a}}^{2}}=\frac{1-\hat{\mathrm{h}}_{\mathrm{a}}^{2}-\hat{\mathrm{c}}_{\mathrm{fam}}^{2}-\hat{\mathrm{c}}_{\mathrm{bloc}}^{2}}{\hat{\mathrm{h}}_{\mathrm{a}}^{2}} ; \lambda_{2}=\frac{\hat{\sigma}_{\mathrm{e}}^{2}}{\hat{\sigma}_{\mathrm{f}}^{2}}=\frac{1-\hat{\mathrm{h}}_{\mathrm{a}}^{2}-\hat{\mathrm{c}}_{\mathrm{fam}}^{2}-\hat{\mathrm{c}}_{\mathrm{bloc}}^{2}}{\hat{\mathrm{c}}_{\mathrm{fam}}^{2}} ; \lambda_{3}=\frac{\hat{\sigma}_{\mathrm{e}}^{2}}{\hat{\sigma}_{\mathrm{b}}^{2}}=\frac{1-\hat{\mathrm{h}}_{\mathrm{a}}^{2}-\hat{\mathrm{c}}_{\mathrm{fam}}^{2}-\hat{\mathrm{c}}_{\mathrm{bloc}}^{2}}{\hat{\mathrm{c}}_{\mathrm{bloc}}^{2}}
$$

Such that,

$\widehat{\mathrm{h}}_{\mathrm{a}}^{2}=\frac{\hat{\sigma}_{\mathrm{a}}^{2}}{\hat{\sigma}_{\mathrm{a}}^{2}+\hat{\sigma}_{\text {fam }}^{2}+\hat{\sigma}_{\text {bloc }}^{2}+\hat{\sigma}_{\mathrm{e}}^{2}}$ individual heritability, in the narrow sense;

$\hat{\mathrm{c}}_{\text {fam }}^{2}=\frac{\hat{\sigma}_{\text {fam }}^{2}}{\hat{\sigma}_{\mathrm{a}}^{2}+\hat{\sigma}_{\text {fam }}^{2}+\hat{\sigma}_{\text {bloc }}^{2}+\hat{\sigma}_{\mathrm{e}}^{2}}$ : coefficient of determination of the effects of specific combining ability (SCC) or the familial dominance effects of sibling species;

$\hat{\mathrm{c}}_{\mathrm{bloc}}^{2}=\frac{\hat{\sigma}_{\text {bloc }}^{2}}{\hat{\sigma}_{\mathrm{a}}^{2}+\hat{\sigma}_{\text {fam }}^{2}+\hat{\sigma}_{\text {bloc }}^{2}+\hat{\sigma}_{\mathrm{e}}^{2}}$; coefficient of determination of the block effects;

$\hat{\sigma}_{\mathrm{a}}^{2}$ : additive genetic variance estimator;

$\hat{\sigma}_{\text {fam }}^{2}$ : SCC variance or genetic variance estimator among families of sibling species;

$\hat{\sigma}_{\text {bloc }}^{2}$ : variance estimator between blocks;

$\hat{\sigma}_{\mathrm{e}}^{2}$ : error or residual variance estimator.

The iterative estimators were obtained to calculate the estimates of the variance components by the REML methodology, by using the EM algorithm (Expectation-Maximization), where, 


$$
\begin{gathered}
\hat{\sigma}_{e}^{2}=\frac{\left(y^{\prime} y-\hat{\mathrm{r}}^{\prime} X^{\prime} y-\hat{a}^{\prime} Z^{\prime} y-\hat{f}^{\prime} W^{\prime} y-\hat{b}^{\prime} S^{\prime} y\right)}{N-r(x)} \\
\hat{\sigma}_{a}^{2}=\frac{\left[\hat{a}^{\prime} A^{-1} \hat{a}+\hat{\sigma}_{e}^{2} \operatorname{tr}\left(A^{-1} C^{22}\right)\right]}{q} \\
\hat{\sigma}_{\text {fam }}^{2}=\frac{\left[\hat{f} \hat{f}+\hat{\sigma}_{e}^{2} \operatorname{tr}\left(C^{33}\right)\right]}{s_{1}} ; \hat{\sigma}_{b l o c}^{2}=\frac{\left[\hat{b} \hat{b}+\hat{\sigma}_{e}^{2} \operatorname{tr}\left(C^{44}\right)\right]}{s_{2}}
\end{gathered}
$$

$\mathrm{C}^{22}, \mathrm{C}^{33}$ and $\mathrm{C}^{44}$ derive from $\mathrm{C}^{-1}$ :

$$
C^{-1}=\left[\begin{array}{llll}
C_{11} & C_{12} & C_{13} & C_{14} \\
C_{21} & C_{22} & C_{23} & C_{24} \\
C_{31} & C_{32} & C_{33} & C_{34} \\
C_{41} & C_{42} & C_{43} & C_{44}
\end{array}\right]^{-1}=\left[\begin{array}{llll}
C^{11} & C^{12} & C^{13} & C^{14} \\
C^{21} & C^{22} & C^{23} & C^{24} \\
C^{31} & C^{32} & C^{33} & C^{34} \\
C^{41} & C^{42} & C^{43} & C^{44}
\end{array}\right]
$$

where, $\mathrm{C}$ : is the coefficient matrix of the model equations; tr: trace matrix; $\mathrm{r}(\mathrm{x})$ : rank of matrix $\mathrm{X}$; $\mathrm{N}$ : total number of data; q: total number of genitors; S1 and S2: total number of crosses and total blocks, respectively.

Mean family heritability was estimated by:

$$
\hat{\mathrm{h}}_{\mathrm{fam}}^{2}=\frac{\hat{\sigma}_{\mathrm{p}}^{2}}{\left\{\left[\hat{\sigma}_{\mathrm{e}}^{2}\left(\frac{\hat{\sigma}_{\mathrm{a}}^{2}}{2}\right)\right]^{\left.\mathrm{n}^{\circ} \mathrm{rep}+\hat{\sigma}_{\mathrm{p}}^{2}\right\}}\right.}
$$

The genotypic variance among families is given by the formula:

$$
\hat{\sigma}_{\mathrm{p}}^{2}=\left(\frac{\hat{\sigma}_{\mathrm{a}}^{2}}{2}\right)+\hat{\sigma}_{\text {bloc }}^{2}
$$

Variance of prediction error of genotypic values (PEV):

$$
(\mathrm{PEV})=\left(1-\hat{\mathrm{h}}_{\mathrm{mf}}^{2}\right) \hat{\sigma}_{\mathrm{p}}^{2}
$$

Predicted genotypic standard deviation (SEP):

$$
(\mathrm{SEP})=\sqrt{\mathrm{PEV}}
$$

Selective accuracy among families:

$$
\mathrm{A}_{\mathrm{c}_{\mathrm{fam}}}=\sqrt{\hat{\mathrm{h}}_{\mathrm{fam}}^{2}}
$$

Coefficient of genetic variation (CVgi\%):

$$
\mathrm{CV}_{\mathrm{gi}} \%=\left(\frac{\hat{\sigma}_{\mathrm{p}}^{2}}{\mu_{\mathrm{g}}}\right) \times 100
$$

where, $\mu_{\mathrm{g}}$ : general mean.

Coefficient of environmental variation $\left(\mathrm{CV}_{\mathrm{e}} \%\right)$ :

$$
\mathrm{CV}_{\mathrm{e}} \%=\left[\frac{\sqrt{\hat{\sigma}_{\mathrm{e}}^{2}+\left(\hat{\sigma}_{\mathrm{a}}^{2} / 2\right)}}{\mu_{\mathrm{g}}}\right] \times 100
$$

Relative variation coefficient $\left(\mathrm{CV}_{\mathrm{r}}\right)$ :

$$
\mathrm{CV}_{\mathrm{r}}=\frac{\mathrm{CV}_{\mathrm{gi}} \%}{\mathrm{CV}_{\mathrm{e}} \%}
$$

\section{Results and Discussion}

The genetic parameters estimated by model 35 for the evaluation of 78 sugarcane families in the present study are presented in Table 2. 
Table 2. Estimated variance components and genetic parameters for the following variables: total plot mass (MTT), mean mass of 1 tiller in the plot (M1C), height (EST) and mean number of canes per square meter (NCM) of the 78 series RB05 sibling species Saccharum spp. families, Paranavaí municipality, Paraná, Brazil

\begin{tabular}{lllll}
\hline Estimators & MTT $(\mathrm{kg})$ & M1C $(\mathrm{kg})$ & EST $(\mathrm{m})$ & NCM \\
\hline$\hat{\sigma}_{\mathrm{a}}^{2}$ & 2.1328 & 0.2470 & 0.0015 & 0.0116 \\
$\hat{\sigma}_{\text {bloc }}^{2}$ & 0.0067 & 0.0160 & 0.0051 & 0.0134 \\
$\hat{\sigma}_{\text {fam }}^{2}$ & 11.7617 & 0.0001 & 0.0186 & 0.0369 \\
$\hat{\sigma}_{\mathrm{e}}^{2}$ & 15.1174 & 0.1250 & 0.0850 & 0.0786 \\
$\hat{\mathrm{h}}_{\mathrm{a}}^{2}$ & $0.0735 \pm 0.0058$ & $0.6364 \pm 0.0701$ & $0.0136 \pm 0.0108$ & $0.0824 \pm 0.0108$ \\
$\hat{\mathrm{c}}_{\text {bloc }}^{2}$ & 0.0002 & 0.0411 & 0.0463 & 0.0952 \\
$\hat{\mathrm{c}}_{\text {fam }}^{2}$ & 0.4053 & 0.0003 & 0.1688 & 0.2626 \\
$\hat{\mathrm{h}}_{\text {fam }}^{2}$ & 0.7985 & 0.7133 & 0.5307 & 0.7166 \\
$\mu_{\mathrm{g}}$ & 6.7493 & 0.7672 & 2.1624 & 1.3927 \\
\hdashline$\hat{\sigma}_{\mathrm{p}}^{2}$ & 12.8281 & 0.1236 & 0.0194 & 0.0427 \\
$\mathrm{PEV}$ & 1.8857 & 0.0354 & 0.0091 & 0.0121 \\
$\mathrm{SEP}$ & 1.3732 & 0.1883 & 0.0954 & 0.1100 \\
$\mathrm{~A}_{\text {ffam }}$ & 0.9236 & 0.8445 & 0.7281 & 0.8465 \\
$\mathrm{CV}_{\mathrm{gi}} \%$ & 53.0667 & 45.8248 & 6.4329 & 14.8350 \\
$\mathrm{CV}_{\mathrm{e}} \%$ & 57.6082 & 46.2308 & 13.5028 & 20.2205 \\
$\mathrm{CV}_{\mathrm{r}}$ & 0.9212 & 0.9912 & 0.4764 & 0.7337 \\
\hline
\end{tabular}

Note. ( $\hat{\sigma}_{\mathrm{a}}^{2}$ : additive genetic variance), $\left(\hat{\sigma}_{\mathrm{b}}^{2}\right.$ : variance between blocks), ( $\hat{\sigma}_{\text {fam }}^{2}$ SCC variance or genetic variance of dominance between families), ( $\hat{\sigma}_{\mathrm{e}}^{2}$ : residual variance), $\left(\hat{\sigma}_{\mathrm{f}}^{2}\right.$ individual phenotypic variance), $\hat{\mathrm{h}}_{\mathrm{a}}^{2}$ : heritability in the narrow sense in the block, i.e., of the additive effects), ( $\hat{\mathrm{h}}_{\mathrm{g}}^{2}$ : heritability in the broad sense, i.e., of the total genotypic effects), ( $\hat{\mathrm{c}}_{\mathrm{bloc}}^{2}$ :coefficient of determination of the effects of blocks), ( $\hat{\mathrm{c}}_{\mathrm{fam}}^{2}$ : coefficient of determination of SCC effects).

According to the classification proposed by Resende (2002), heritability can be considered as low magnitude when $\widehat{\mathrm{h}}_{\mathrm{a}}^{2}<0.15$, medium magnitude when $0.15<\widehat{\mathrm{h}}_{\mathrm{a}}^{2}<0.50$ and high magnitude when $\widehat{\mathrm{h}}_{\mathrm{a}}^{2}>0.50$. Thus, MTT and M1C responses evaluated herein presented high heritability, while EST and NCM presented very low heritability, demonstrating that the latter two are highly influenced by the environment. EST presented average heritability among high-magnitude sugarcane families, suggesting an inter-family genetic difference regarding this characteristic, with no difference observed between individuals from the same family.

One likely explanation for this behavior is due to the low number of replicates in the experiment, as, even though each family had been distributed five times in the field, some plants throughout the cropping cycle did not sprout after the first cut or simply did not develop to the point to be able to generate any nonzero data. Another explanation for the event is precisely due to the nature of the Federer blocks design, (Federer, 1956), where most of the treatment and block effects are assumed to be fixed when adopting the vector $\mathrm{r}$ as random (due to the unbalance of family repetitions), leading to a random model. Consequently the effects of repetitions would be distributed confusingly within the block effects, which, in turn, could erroneously inflate the non-additive genetic variance portion.

The high genetic variability observed for MTT, is, in part, due to the high magnitude of additive variance, making this variable promising when performing family selection, since this characteristic can probably be passed on to the next generation.

In sugarcane populations, both dominance (non-additive) and additive effects depend, mainly, on allele frequency, complementarity and genetic divergence (Barbosa, Resende, Bressiani, Silveira, \& Peternelli, 2005).

With the exception of EST, all the evaluated variables were explained by the additive genetic variance portion, corroborating with other studies (Bastos, 2003; Barbosa et al., 2005; Silva et al., 2015).

The high genetic variation coefficients for MTT and M1C (319\% and 24\%, respectively) suggest that sugarcane families can be selected for these characteristics, as a significant genetic variability among the evaluated families exist. This was not observed at the same intensity for EST and NCM, explained, probably, by the fact that crosses were carried out between parents with a very narrow genetic base between both EST and NCM, that is, very close, corroborating previous studies (Daniels \& Roach, 1987; Jackson, 2005; Silva, Vidigal, Vidigal Filho, Scapim, Daros, \& Silvério, 2005). 
Since values for all study variables allowed for family selection, MTT, presented a high genotypic value for the first five families (F75M70, F60M19, F41M82, F78M45 and F66M30), if selecting only these five families. On average, MTT would be increased by $82.11 \%$ in the next stages of the breeding program. The criterion for judging a family as "better" is observed when the relative means (\%) generated by their selection adds up to values other than zero. The opposite is also true; families with relative genotypic values $(\mathrm{Vgc}) \leq 0$ were disregarded. Thus Table 3 lists only the best families. According to this data, the first 34 families could be used regarding MTT, since a relative gain in family selection of $31.76 \%$ (means of the 34 best families) would be observed in relation to the experimental means $(5.9044 \mathrm{~kg})$.

Table 3. Genotypic values (Vgc) and general means of the 78 Saccharum spp. sibling species series RB05 families for total mass of the plot (MTT), average mass of 1 tiller in plot (M1C), height (EST) and mean number of stems per square meter (NCM), Paranavaí municipality, Paraná, Brazil

\begin{tabular}{|c|c|c|c|c|c|c|c|c|}
\hline \multirow{2}{*}{ Class $^{1}$} & \multicolumn{2}{|c|}{ MTT (Kg) } & \multicolumn{2}{|c|}{$\mathrm{M} 1 \mathrm{C}(\mathrm{Kg})$} & \multicolumn{2}{|c|}{ EST (m) } & \multicolumn{2}{|c|}{$\mathrm{NCM}$} \\
\hline & Family & Vgc & Family & Vgc & Família & Vgc & Family & Vgc \\
\hline 1 & F57M46 & 9.1064 & F04M14 & 0.9636 & F57M46 & 3.0511 & F75M70 & 1.7559 \\
\hline 2 & F75M70 & 9.0272 & F05M68 & 0.9594 & F60M16 & 3.0358 & F47M31 & 1.6977 \\
\hline 3 & F47M31 & 8.9789 & F62M35 & 0.9477 & F34M02 & 3.0245 & F41M64 & 1.6801 \\
\hline 4 & F60M19 & 8.8532 & F10M29 & 0.9462 & F60M19 & 2.9833 & F57M46 & 1.6414 \\
\hline 5 & F66M30 & 8.6201 & F13M17 & 0.9458 & F60M43 & 2.8512 & F66M30 & 1.6185 \\
\hline 6 & F78M45 & 8.3717 & F60M19 & 0.9421 & F47M31 & 2.8425 & F35M06 & 1.6094 \\
\hline 7 & F04M14 & 8.2547 & F27M56 & 0.9398 & F41M64 & 2.8277 & F60M19 & 1.6067 \\
\hline 8 & F41M64 & 8.2482 & F70M30 & 0.9390 & F78M45 & 2.8125 & F03M33 & 1.5984 \\
\hline 9 & F13M17 & 8.1555 & F41M82 & 0.9366 & F14M08 & 2.8021 & F70M75 & 1.5980 \\
\hline 10 & F41M21 & 8.0775 & F14M08 & 0.9336 & F60M10 & 2.7706 & F23M76 & 1.5828 \\
\hline 11 & F35M06 & 7.8854 & F19M29 & 0.9281 & F66M30 & 2.7462 & $\mathrm{~F} 41 \mathrm{M} 21$ & 1.5798 \\
\hline 12 & F03M33 & 7.7339 & F60M10 & 0.9277 & F75M70 & 2.7453 & F67M37 & 1.5779 \\
\hline 13 & F41M82 & 7.7289 & F15M14 & 0.9274 & F44M02 & 2.7225 & F60M71 & 1.5754 \\
\hline 14 & F34M02 & 7.6205 & F26M65 & 0.9216 & F04M14 & 2.7215 & F17M35 & 1.5468 \\
\hline 15 & F41M48 & 7.5850 & F34M02 & 0.9208 & F41M48 & 2.6796 & F78M45 & 1.5453 \\
\hline 16 & F70M30 & 7.5586 & F66M30 & 0.9044 & F41M47 & 2.6197 & F01M39 & 1.5379 \\
\hline 17 & F30M36 & 7.5067 & $\mathrm{~F} 82 \mathrm{M} 01$ & 0.9042 & F62M35 & 2.5993 & F56M70 & 1.5271 \\
\hline 18 & F46M07 & 7.4973 & F57M46 & 0.8903 & $\mathrm{~F} 41 \mathrm{M} 22$ & 2.5906 & F44M02 & 1.5270 \\
\hline 19 & F60M43 & 7.4963 & F30M36 & 0.8874 & F61M38 & 2.5115 & F70M30 & 1.5256 \\
\hline 20 & F60M71 & 7.4892 & F70M48 & 0.8873 & F82M01 & 2.4569 & F60M43 & 1.5190 \\
\hline 21 & F82M01 & 7.2387 & F17M25 & 0.8830 & $\mathrm{~F} 41 \mathrm{M} 21$ & 2.4346 & F41M48 & 1.5137 \\
\hline 22 & F57M12 & 7.1862 & F60M16 & 0.8767 & F41M60 & 2.4286 & F46M07 & 1.5089 \\
\hline 23 & F11M69 & 7.1567 & F75M70 & 0.8670 & F41M53 & 2.4243 & F57M12 & 1.4972 \\
\hline 24 & F17M35 & 7.1389 & F78M45 & 0.8655 & F56M70 & 2.4170 & F04M14 & 1.4686 \\
\hline 25 & F27M56 & 7.1022 & F47M31 & 0.8643 & F01M24 & 2.4120 & F17M25 & 1.4684 \\
\hline 26 & F44M02 & 7.0406 & F10M51 & 0.8614 & F70M48 & 2.4071 & F48M41 & 1.4652 \\
\hline 27 & F56M70 & 7.0324 & F41M48 & 0.8537 & F70M30 & 2.4005 & F41M20 & 1.4610 \\
\hline 28 & F67M37 & 7.0196 & F26M55 & 0.8433 & F15M14 & 2.3767 & F41M82 & 1.4454 \\
\hline 29 & F01M39 & 6.9768 & $\mathrm{~F} 41 \mathrm{M} 21$ & 0.8329 & F57M12 & 2.3557 & F82M01 & 1.4452 \\
\hline 30 & F23M76 & 6.9564 & F54M07 & 0.8258 & F54M07 & 2.3409 & F11M69 & 1.4245 \\
\hline 31 & F60M16 & 6.9543 & F57M12 & 0.8114 & F26M65 & 2.3092 & F41M60 & 1.4234 \\
\hline 32 & F15M14 & 6.9477 & F14M82 & 0.8076 & F70M56 & 2.2832 & F04M40 & 1.4174 \\
\hline 33 & F05M68 & 6.8980 & F41M60 & 0.7997 & F14M22 & 2.2669 & F48M70 & 1.4166 \\
\hline 34 & F26M65 & 6.8394 & F41M22 & 0.7937 & F67M37 & 2.2364 & F59M50 & 1.4030 \\
\hline 35 & F62M35 & 6.8262 & F01M24 & 0.7924 & F60M52 & 2.2128 & F60M52 & 1.3998 \\
\hline 36 & F17M25 & 6.7787 & F41M64 & 0.7783 & F02M77 & 2.1969 & F41M53 & 1.3992 \\
\hline 37 & F70M75 & 6.7738 & F46M07 & 0.7754 & F40M74 & $\dagger$ & F45M78 & 1.3986 \\
\hline 38 & F04M40 & $\dagger$ & F81M02 & $\dagger$ & F30M36 & $\dagger$ & F70M56 & 1.3979 \\
\hline Mean & & 6.7493 & & 0.7672 & & 2.1624 & & 1.3927 \\
\hline
\end{tabular}

Note. ${ }^{1}$ Classification; $\uparrow$ : Disregarded values, since they were below the respective experimental means. 
The M1C for the first five families (F04M14, F05M68, F62M35, F27M56 and F70M30) would increase in $42.58 \%$ in the next stages of the breeding program, and the relative means of the 37 best families would increase in $15.17 \%$ compared to the experimental means $(0.5916 \mathrm{~kg})$. For EST, an increase of approximately $14.87 \%$ for the the five best families (F57M46, F60M16, F34M02, F60M19 and F60M43) would be observed in the next stages of the program, with a relative means increase of $6.02 \%$, for the 38 best families compared to the experimental average $(0.5916 \mathrm{~m})$. Finally, an approximate gain of $21.96 \%$ would be observed by using the first five families (F75M70, F47M31, F41M64, F57M46 and F35M06), with a relative means increase of 9.77\% for the 36 best families compared to the experimental average (1.3938).

All four evaluated characteristics led to the selection of 34, 37, 38 and 36 families, respectively, representing $43.59 \%, 47.44 \%, 48.72 \%$ and $46.15 \%$ of the evaluated family means, within the means of the four evaluated variables, of $46.48 \%$, of the RB05 series families, corroborating previous studies (Cox, Hogarth, \& Smith, 2000; Silva et al., 2015).

\section{Conclusions}

The evaluated variables (MTT, M1C, EST and NCM) were susceptible between medium and high magnitude concerning family selection accuracy, with values ranging between 0.4675 and 0.8446 .

Families were selected allowing for increases in the four evaluated variables for the next phases of the breeding program.

The genetic variations for MTT and M1C were explained by the additive genetic portion, while much of this explanation was due to the non-additive portion for EST and NCM.

The REML/BLUP methodology is important in the selection of sugarcane families in unbalanced experiments, revealing information from genetic parameters estimates to the breeder, to more accurately guide the next stages of the crop breeding program.

\section{References}

Almeida, L. M., Viana, A. P., Amaral Júnior, A. T., \& Júnior, J. B. C. (2014). Breeding full-sib families of sugar cane using selection index. Cienc. Rural, 44(4), 605-611. https://doi.org/10.1590/S0103-847820140004 00005

Almeida Filho, J. E., Tardin, F. D., Guimarães, J. F., \& Resende, M. D. (2016). Multi-trait BLUP model indicates sorghum hybrids with genetic potential for agronomic and nutritional traits. Genet. Mol. Res., 15(1), 1-9. https://doi.org/10.4238/ gmr.15017071

Atkin, F. C.; Dieters, M. J., \& Stringer, J. K. (2009). Impact of depth of pedigree and inclusion of historical data on the estimation of additive variance and breeding valuesin a sugarcane breeding program. Theor. Appl. Genet., 119(3), 555-565. https://doi.org/10.1007/s00122-009-1065-7

Barbosa, M. H. P., Resende, M. D. V., Bressiani, J. A., Silveira, L. C. I., \& Peternelli, L. A. (2005). Selection of sugarcane families and parents by Reml/Blup. Crop Breeding and Applied Biotechnology, 5, 443-450. https://doi.org/10.12702/1984-7033.v05n04a10

Bastos, I. T., Barbosa, M. H. P., Cruz, C. D., Burnquist, W. L., Bressiani, J. A., \& Silva, F. L. (2003). Análise dialélica em clones de cana-de-açúcar. Bragantia, 62, 199-206. https://doi.org/10.1590/S0006-87052003 000200004

Brasileiro, B. P., Paula Mendes, T. O., Peternelli, L. A., Silveira, L. C. I. da, Resende, M. D. V. de, \& Barbosa, M. H. P. (2016). Simulated Individual Best Linear Unbiased Prediction versus Mass Selection in Sugarcane Families. Crop Sci., 56, 570-575. https://doi.org/10.2135/cropsci2015.03.0199

Castro, R. D., Peternelli, L. A., Resende, M. D. V., Marinho, C. D., Costa, P. M. A., Barbosa, M. H. P., \& Moreira, E. F. A. (2016). Selection between and within full-sib sugarcane families using the modified BLUPIS method (BLUPISM). Genet. Mol. Res., 15(1), 1-10. https://doi.org/10.4238/gmr.15017334

Cox, M.; Hogarth, M., \& Smith, G. (2000). Cane breeding and improvement. In M. Hogarth, \& P. Allsopp (Eds.), Manual of cane growing (pp. 91-108). Brisbane, Australian.

Daniels, J., \& Roach, B. T. (1987). Taxonomy and evolution. In D. J. Heinz (Ed.), Sugarcane Improvement Trought Breeding (pp. 7-84). Amsterdam, Holland. https://doi.org/10.1016/B978-0-444-42769-4.50007-2

Dillewijn, C. V. (1952). Botany of sugarcane. The Chronica Botanica: Waltham.

Federer, W. T. (1956). Augmented (or Hoonuiaku) designs. Hawaiian Planters Record, 55(2), 191-208. 
Jackson, P. A. (2005). Breeding for improved sugar content in sugarcane. Field Crops Research, 92, 277-290. https://doi.org/10.1016/j.fcr.2005.01.024

Kennedy, B. W., \& Sorensen, D. A. (1988). Properties of mixed-model methods for prediction of genetic merit. In B. S. Weir, E. J. Eisen, M. M. Goodman, \& G. Namkoog (Eds.), Second international conference on quantitative genetics (pp. 91-103). Sunderland, England.

Meyer, K. (1991). Estimating variances and covariances for multivariateanimal models by restricted maximum likelihood. Genet Selection Evolucion, 23, 67-83. https://dx.doi.org/10.1186\%2F1297-9686-23-1-67

Oakey, H., Verbyla, A., Pitchford, W., Cullis, B. R., \& Kuchel, H. (2006). Jointmodeling of additive and non-additive genetic line effects insingle field trials. Theoretical and Applied Genetics, 113, 809-819. https://doi.org/10.1007/s00122-006-0333-z

Oakey, H., Verbyla, A. P., Cullis, B. R., Wei, X., \& Pitchford, W. S. (2007). Joint modeling of additive and non-additive (genetic line) effects inmulti-environment trials. Theoretical and Applied Genetics, 114, 1319-1332. https://doi.org/10.1007/s00122-007-0515-3

Oliveira, R. A. de., Daros, E., Bespalhok Filho, J. C., Zambon, J. L. C., Ido, O. T., Weber, H., Resende, M. D. V. de, \& Zeni Neto, H. (2008). Seleção de famílias de cana-de-açúcar via modelos mistos. Scientia Agraria, 9(3), 269-274. https://doi.org/10.5380/rsa.v9i3.11564

Pedroso, C. A., Benites, F. R. G., Barbosa, M. H. P., Resende, M. D. V., \& Silva, F. L. (2009). Eficiência de Índices de seleção utilizando a metodologia REML/BLUP no melhoramento da cana-de-açúcar. Scientia Agraria, 10, 31-36. https://doi.org/10.5380/rsa.v10i1.11711

Resende, M. D. V. de (2002). Genetics, biometrics and statistics in breeding perennial plants. Brasília, Brazil: Embrapa Informação Tecnológica.

Resende, M. D. V. de (2007). Software SELEGEN-REML/BLUP: Statistical system and genetic screening computed via mixed linear models. Colombo, Paraná: Embrapa Florestas.

Silva, G. M., Vidigal M. C. G., Vidigal Filho, P. S., Scapim, C. A., Daros, E., \& Silvério, L. (2005). Genetic diversity among sugarcane clones (Saccharum spp.). Acta Scientiarum Agronomy, 27, 315-319. https://doi.org/10.4025/actasciagron.v27i2.1851

Silva, F. L. da, Barbosa, M. H. P., Resende, M. D. V. de, Peternelli, L. A., \& Pedrozo, C. A. (2015). Efficiency of selection within sugarcane families via simulated individual BLUP. Crop Breeding and Applied Biotechnology, 15, 1-9. https://doi.org/10.1590/1984-70332015v15n1a1

\section{Copyrights}

Copyright for this article is retained by the author(s), with first publication rights granted to the journal.

This is an open-access article distributed under the terms and conditions of the Creative Commons Attribution license (http://creativecommons.org/licenses/by/4.0/). 